\title{
COVID-19: guidance on palliative care from a European Respiratory Society international task force
}

\author{
Daisy J.A. Janssen (101,2, Magnus Ekström $\mathbb{1}^{3,4}$, David C. Currow ${ }^{4,5}$, \\ Miriam J. Johnson ${ }^{5}$, Matthew Maddocks ${ }^{6}$, Anita K. Simonds ${ }^{7}$, \\ Thomy Tonia ${ }^{8}$ and Kristoffer Marsaa ${ }^{9}$
}

Affiliations: ${ }^{1}$ Dept of Research and Development, CIRO, Horn, The Netherlands. ${ }^{2}$ Dept of Health Services Research, Care and Public Health Research Institute, Faculty of Health Medicine and Life Sciences, Maastricht University, Maastricht, The Netherlands. ${ }^{3}$ Faculty of Medicine, Dept of Clinical Sciences Lund, Respiratory Medicine and Allergology, Lund University, Lund, Sweden. ${ }^{4}$ IMPACCT, Faculty of Health, University of Technology Sydney, Ultimo, Sydney, Australia. ${ }^{5}$ Wolfson Palliative Care Research Centre, University of Hull, Hull, UK. 'Cicely Saunders Institute of Palliative Care, Policy and Rehabilitation, King's College London, London, UK. ${ }^{7}$ Sleep and Ventilation Unit, Royal Brompton and Harefield NHS Foundation Trust, London, UK. ${ }^{8}$ Institute of Social and Preventive Medicine, University of Bern, Bern, Switzerland. ${ }^{9}$ Palliative Unit, Herlev and Gentofte Hospital, Copenhagen, Denmark.

Correspondence: Daisy J.A. Janssen, Dept of Research and Development, CIRO, Hornerheide 1, 6085 NM, Horn, The Netherlands. E-mail: daisyjanssendciro-horn.nl

@ERSpublications

This multi-national task force provides consensus recommendations for palliative care for patients with COVID-19 https://bit.ly/31X83oZ

Cite this article as: Janssen DJA, Ekström M, Currow DC, et al. COVID-19: guidance on palliative care from a European Respiratory Society international task force. Eur Respir J 2020; 56: 2002583 [https://doi. org/10.1183/13993003.02583-2020].

\section{ABSTRACT}

Background: Many people are dying from coronavirus disease 2019 (COVID-19), but consensus guidance on palliative care in COVID-19 is lacking. This new life-threatening disease has put healthcare systems under pressure, with the increased need of palliative care provided to many patients by clinicians who have limited prior experience in this field. Therefore, we aimed to make consensus recommendations for palliative care for patients with COVID-19 using the Convergence of Opinion on Recommendations and Evidence (CORE) process.

Methods: We invited 90 international experts to complete an online survey including stating their agreement, or not, with 14 potential recommendations. At least $70 \%$ agreement on directionality was needed to provide consensus recommendations. If consensus was not achieved on the first round, a second round was conducted.

Results: 68 (75.6\%) experts responded in the first round. Most participants were experts in palliative care, respiratory medicine or critical care medicine. In the first round, consensus was achieved on 13 recommendations based upon indirect evidence and clinical experience. In the second round, 58 (85.3\%) out of 68 of the first-round experts responded, resulting in consensus for the 14th recommendation.

Conclusion: This multi-national task force provides consensus recommendations for palliative care for patients with COVID-19 concerning: advance care planning; (pharmacological) palliative treatment of breathlessness; clinician-patient communication; remote clinician-family communication; palliative care involvement in patients with serious COVID-19; spiritual care; psychosocial care; and bereavement care. Future studies are needed to generate empirical evidence for these recommendations. 


\section{Introduction}

As of 30 June 2020, 10360882 coronavirus disease 2019 (COVID-19) cases had been confirmed with 507014 deaths due to COVID-19 recorded globally [1]. However, both the number of confirmed cases and COVID-19-related mortality are likely to be underestimated as not all cases and deaths due to COVID-19 are confirmed or recorded [2]. Patients may die at home, in the hospital, or in other facilities.

The importance of palliative care in this COVID-19 pandemic has been acknowledged $[3,4]$. Despite its paramount need, providing high-quality palliative care during this pandemic is challenging. Patients may deteriorate quickly, healthcare resources are under pressure, isolation is required and family visits are restricted [5]. A case series of 101 hospitalised patients with COVID-19 referred for palliative care showed that most patients died within 3 days [6], and the need for "emergency" palliative care in COVID-19 has been suggested [7]. Worries have arisen about limited access to palliative care because of high demands worldwide in the pandemic [3]. Despite a number of guidelines and resources proposed by professional and other bodies [8,9], neither evidence- nor consensus-based guidelines about palliative care in COVID-19 are available. In fact, a survey among hospices in Italy revealed that healthcare professionals lacked guidance on care for people dying from COVID-19 [10]. The COVID-19 crisis has been a professionally and personally challenging period for healthcare workers. Although the underlying principles of palliative care have not changed, the specific challenges of COVID-19 require specific guidance [4]. Therefore, the aim of the current study was to develop guidance on palliative care in COVID-19 patients through consensus, pending empirical evidence.

\section{Methods}

We conducted a survey following the Convergence of Opinion on Recommendations and Evidence (CORE) process. The CORE process is a consensus-based approach to making clinical recommendations, which has been shown to yield recommendations that are concordant with those developed using Institute of Medicine-adherent methodology [11].

An ad hoc international task force was assembled, including European Respiratory Society (ERS) key opinion leaders in the field of palliative care and respiratory medicine. Invitations were sent to 90 experts (in palliative care, respiratory medicine, clinical care and research) identified by the task force members. A survey was created using SurveyMonkey software (SurveyMonkey, San Mateo, CA, USA), consisting of 14 questions, with the aim of providing consensus recommendations for clinical care. Each question consisted of three parts. The first part presented the question in a modified PICO (Patient, Intervention, Comparator, Outcomes) format. The second part was a multiple-choice question where the participant was asked to choose a recommendation for or against a given therapy: strong recommendation for; conditional recommendation for; no recommendation for or against; conditional recommendation against; or strong recommendation against an intervention (table 1). The third part was a free text box for comments. Three demographic questions were asked about each participant's country, profession and field of expertise.

In the survey, we used the term "serious COVID-19", defined as COVID-19 that carries a high risk of mortality, negatively impacts quality of life and daily function, and/or is burdensome in symptom, treatment or caregiver stress. This definition was based on that of serious illness on Pallipedia [12].

The survey was administered from 2 to 11 June 2020. Several reminders were sent. Agreement of directionality was tabulated for each multiple-choice question. A priori, we defined that $\geqslant 70 \%$ agreement on directionality (agreement or disagreement) was needed to be able to provide consensus recommendations [13]. The proportion of respondents per choice per question was calculated and expressed as a percentage of the total number of respondents. A second round was conducted from 15 to

TABLE 1 Definitions of recommendations

\section{Strong recommendation for an intervention}

Conditional recommendation for an intervention
Should be chosen when experts were certain that the desirable consequences outweigh the undesirable consequences lor the converse for recommendation against). A strong recommendation is one that most well-informed patients would follow.

Should be chosen when experts were uncertain that the desirable consequences of the intervention outweigh the undesirable consequences (or the converse, for recommendation against). A conditional recommendation indicates that well-informed patients may make different choices regarding whether to have or not have the intervention. 
18 June 2020 for the question not leading to consensus in the first round. Results of the first round were summarised for the second round. Free text comments were summarised by Dr D.J.A. Janssen and these summaries were checked with the data by Dr K. Marsaa.

\section{Results}

Respondents

In the first round, 68 (75.6\%) out of 90 invited experts participated. Respondents were from 15 countries (Australia, and countries in Europe and North America) and most were physicians (table 2). Two-thirds had a background in palliative care and one half in respiratory medicine. The second round was completed by $58(85.3 \%)$ of the 68 previous respondents.

\section{Recommendations}

The 14 recommendations for palliative treatment and care are shown below. For each recommendation, a theoretical rationale is provided, followed by the results of the present survey. For some recommendations, barriers or concerns for implementation were mentioned (table 3).

1) Advance care planning (discussion of goals and preferences for future medical treatment and carel should be routinely performed or reviewed by clinicians with patients and their loved ones at diagnosis of serious COVID-19

Rationale

Advance care planning (ACP) enables individuals to define goals and preferences for future medical treatment and care, to discuss these goals and preferences with family and healthcare providers, and to record and review these preferences if appropriate [14]. Early ACP discussions at the time of hospitalisation are suggested to avoid unwanted and burdensome life-sustaining treatments $[15,16]$.

\section{TABLE 2 Characteristics of respondents}

\section{Characteristic}

Respondents $\mathrm{n}(\%)$

Total
Country
Australia
Austria
Belgium
Canada
Denmark
Germany
Ireland
Italy
The Netherlands
Poland
Portugal
Sweden
Switzerland
UK
USA
Profession ${ }^{\#}$
Physician
Nurse
Allied healthcare professional
Researcher
Expertise
Palliative care
Respiratory medicine
Critical care medicine
Geriatrics
Family medicine
Internal medicine
Other

\footnotetext{
${ }^{\#}$ : respondents could report more than one profession and/or expertise.
} 
TABLE 3 The barriers or concerns about implementation of recommendations reported by experts

\section{Recommendation}

1) ACP should be routinely performed or reviewed by clinicians with patients and their loved ones at diagnosis of serious COVID-19

6) Staff taking care of patients with serious COVID-19 should receive training in optimising clinician-patient communication whilst wearing PPE

7) Staff taking care of patients with serious COVID-19 should receive training in online clinician-family communication (while using telephone or video conferencingl

8) Healthcare professionals trained in providing palliative care should be involved in cases where hospitalised patients with serious COVID-19 have persistent symptoms and concerns despite optimal disease treatment

9) Healthcare professionals trained in providing palliative care should be involved in cases where patients with serious COVID-19 have persistent symptoms and concerns despite optimal disease treatment, and are being treated at home

10) Healthcare professionals providing spiritual care (such as chaplains) should be part of the treatment team of patients with serious COVID-19 with persistent symptoms and concerns despite optimal disease treatment (irrespective of setting, so in the hospital, community or long-term care facilities]

11) Healthcare professionals providing psychosocial care (such as psychologists and social workers) should be part of the treatment team of patients with serious COVID-19 with persistent symptoms and concerns despite optimal disease treatment (irrespective of setting, so in the hospital, community or long-term care facilities)

12) Family members/loved ones should be invited and supported le.g. being provided with PPE if indicated) to visit the dying patient with COVID-19 in person

13) Family members/loved ones of deceased patients with COVID-19 should be offered bereavement support by healthcare professionals trained in palliative care or bereavement support

\section{Barriers/concerns}

The disease evolves rapidly resulting in lack of clarity on the patient's condition preventing a possible long-term plan

The often-rapid trajectory towards death

Patients may be too ill to participate in ACP

Patients may experience too much anxiety to participate in ACP conversations

The family are not physically present

Practical concerns about implementing training during the pandemic

Practical concerns about implementing training during the pandemic

Practical concerns

Resource limitations, including limited availability of palliative care specialists

Limited availability of PPE

Limited resources available for patients at home or in care homes, including limited availability of palliative care specialists

Risk of transmission of COVID-19

Limited availability of PPE

Limited availability of spiritual/existential care providers

Patients being too breathless to talk

Limited availability of PPE

Limited availability of psychosocial healthcare professionals

Risk of transmission of COVID-19 to psychosocial healthcare professionals

Limited availability of PPE

Visits are a source of distress for families and staff Risk of transmission of COVID-19 to visitors

Lack of time between diagnosing dying and actual death

Limited availability of bereavement support

ACP: advance care planning; COVID-19: coronavirus disease 2019; PPE: personal protection equipment.

Most of the experts strongly (67.6\%) or conditionally (29.4\%) recommended that ACP should be routinely conducted or reviewed by clinicians with patients and their loved ones, at the time of diagnosis of serious COVID-19 (figure 1a). 

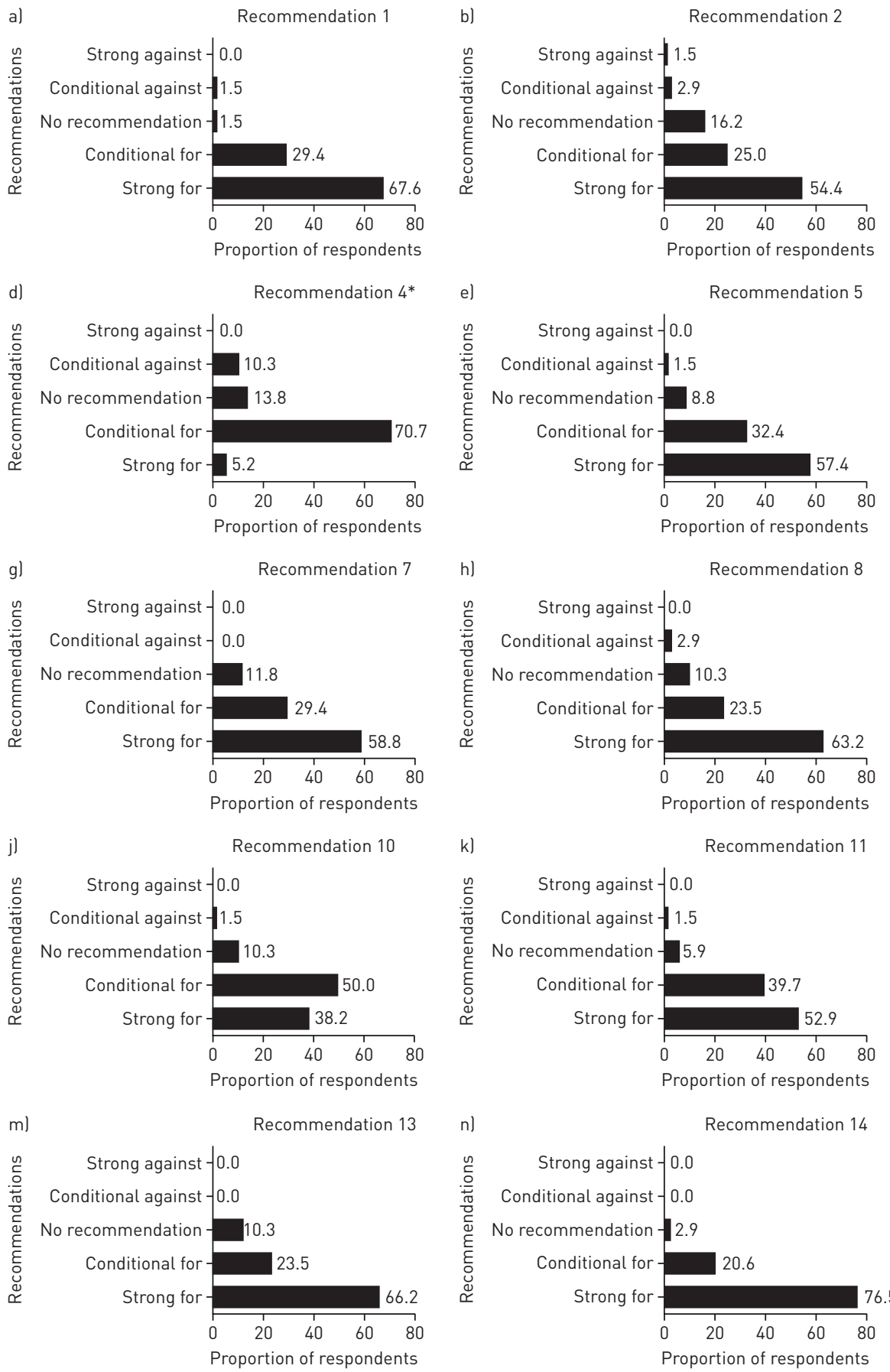

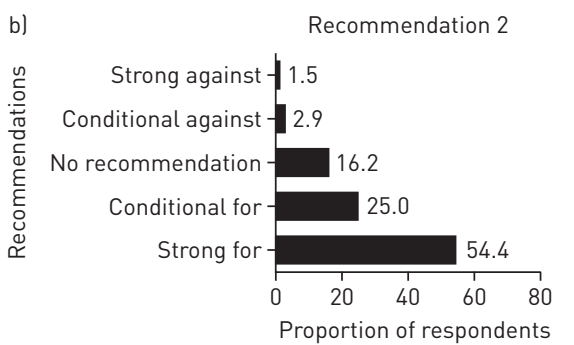

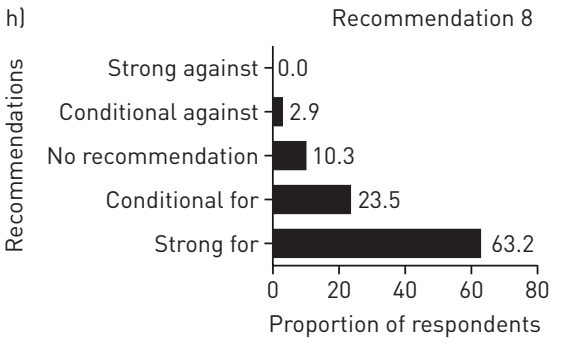
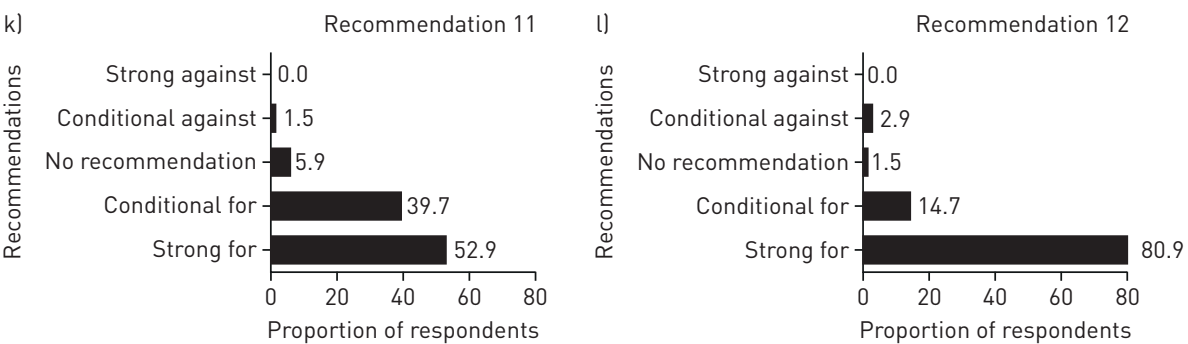

i) Recommendation 9

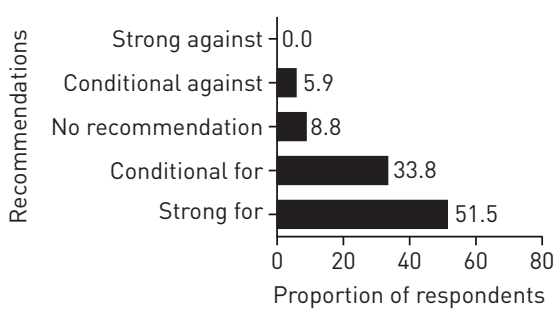

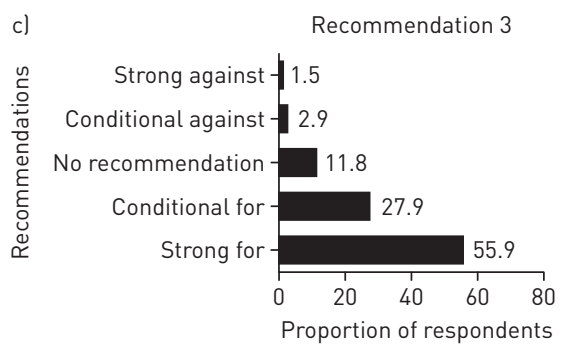

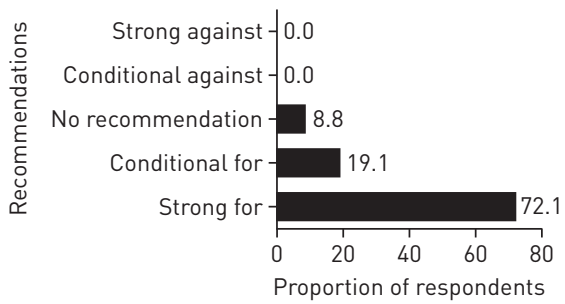

n)

Recommendation 14

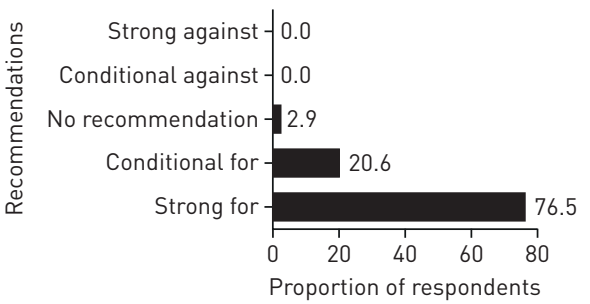

FIGURE 1 Experts' responses to the 14 questions. *: results from the second round.

ACP was mentioned as "the first critical step to guide treatment for seriously ill patients". A few experts suggested that ACP should be offered, but patient wishes should be respected if they declined. Some stated that whether ACP is offered should be dependent on the presence of comorbidities and the likelihood of a poor outcome. The need for and advantages of proactive ACP before the occurrence of COVID-19, especially in frail older patients, were also mentioned.

\section{2) ACP should be re-evaluated prior to discharge of recovered COVID-19 patients from hospital Rationale}

Life-sustaining treatment preferences are likely to change, for example after a change in health status. While some patients are less willing to undergo life-sustaining treatments after a decline in functional 
status, other patients are even more willing to undergo life-sustaining treatments [17]. For deliberate decisions concerning life-sustaining treatments, patients need information about possible outcomes and the likelihood of negative outcomes after treatment [18]. An intensive care unit (ICU) admission for acute respiratory distress syndrome associated with COVID-19 may have a lower chance of survival than an ICU admission for another illness [16]. So, a preference to forego ICU admission in cases of COVID-19 might not result in a decision to forego all ICU admissions. Therefore, evaluation of ACP before discharge of recovered patients with COVID-19 is warranted.

\section{Results}

Most experts strongly (54.4\%) or conditionally (25.0\%) recommended that ACP should be re-evaluated prior to the discharge of recovered COVID-19 patients from hospital (figure 1b).

Experts mentioned that if patients recover, the serious illness might have influenced their preferences regarding life-sustaining treatments. Moreover, patients may be able to more fully participate in these discussions than during the acute illness. It was also suggested that re-evaluation of ACP after discharge by the family physician might be preferable.

\section{3) Patients presenting with serious COVID-19 and distressing breathlessness despite optimal treatment of underlying causes should be given low-dose opioids for the palliative treatment of breathlessness \\ Rationale}

Breathlessness is one of the most prevalent symptoms in hospitalised patients dying from COVID-19 $[6,15,19,20]$. Opioids are widely used in palliative care with an evidence base effect for relieving breathlessness [21]. Nevertheless, there are no reported trials in COVID-19. A first case series of hospitalised patients with COVID-19 referred for palliative care showed that most patients were treated with morphine (median dose $10 \mathrm{mg}$ in $24 \mathrm{~h}$ subcutaneously) and some received fentanyl (median dose $100 \mu \mathrm{g}$ in $24 \mathrm{~h}$ ) or alfentanil (median dose $500 \mu \mathrm{g}$ in $24 \mathrm{~h}$ ) [6]. However, effectiveness was clinician rated and data were not collected for individual drugs. Another case series of 30 hospitalised deceased patients showed that $76.7 \%$ of the patients used intravenous morphine but, again, effectiveness was not evaluated [19].

\section{Results}

Most experts strongly (55.9\%) or conditionally (27.9\%) recommended that patients presenting with serious COVID-19 and distressing breathlessness, despite optimal treatment of underlying causes, should be given low-dose opioids for the palliative treatment of breathlessness (figure 1c).

Experts mentioned the need to treat breathlessness according to current palliative care guidelines, including opioids. They cited positive experiences of opioids for breathlessness in COVID-19. Some experts stated that effectiveness for breathlessness in COVID-19 should be confirmed in future studies, but agreed with the recommendation. Conversely, others cited the lack of evidence of effectiveness for breathlessness in COVID-19 as a reason to disagree or provide no recommendation for or against opioids. Experts stated the need for careful consideration of the individual situation, including the risk of adverse effects.

\section{4) Patients presenting with serious COVID-19 and distressing breathlessness despite optimal treatment of underlying causes should be given benzodiazepines for the palliative treatment of breathlessness \\ Rationale}

Clinical practice statements mention benzodiazepines as palliative treatment of breathlessness [22]. Nevertheless, a Cochrane review showed no evidence for benzodiazepines for breathlessness in patients with cancer or COPD [23]. The review suggested that benzodiazepines could be considered for palliative treatment of breathlessness when non-pharmacological measures and opioids fail, particularly in patients with distress and anxiety [23]. Data on effectiveness for breathlessness in COVID-19 are lacking.

\section{Results}

In the first round, $11.8 \%$ of the respondents gave a strong recommendation for benzodiazepines for distressing breathlessness and $44.1 \%$ gave a conditional recommendation. As the predefined $70 \%$ threshold for consensus was not reached, the question was asked again in second round. In this round, 5.2\% of the respondents gave a strong and $70.7 \%$ of the respondents provided a conditional recommendation for benzodiazepines for distressing breathlessness (figure 1d).

A few experts stressed the fact that there is a lack of evidence of the effect for breathlessness, while there is evidence of side-effects. Several experts mentioned that benzodiazepines should be taken into 
consideration if patients were very distressed or anxious, when opioids didn't have enough effect, when opioids were contra-indicated, or in the last days of life.

5) Patients with serious COVID-19 in palliative care and distressing breathlessness should be given oxygen therapy for the palliative treatment of breathlessness when their transcutaneous oxygen saturation is $<90 \%$

Rationale

Oxygen therapy in patients with COVID-19 and hypoxaemia may help in reducing breathlessness in palliative care [15]. Oxygen therapy improves survival in patients with severe hypoxaemia. The evidence for a symptomatic effect on breathlessness is conflicting, but oxygen may relieve breathlessness in some patients with hypoxaemia [24].

\section{Results}

A great majority gave a strong (57.4\%) or conditional (32.4\%) recommendation for oxygen therapy for the palliative treatment of breathlessness when their transcutaneous oxygen saturation is $<90 \%$ (figure 1e).

Free text comments showed some different opinions. Some stressed the importance of correcting hypoxaemia, without the primary aim of reducing breathlessness. Several suggested threshold oxygen saturations other than $90 \%$, such as $85 \%, 88 \%$ and $92 \%$. Others stressed that supplemental oxygen could be started if patients prefer, but discontinued if breathlessness was not reduced. Some experts highlighted the insufficient evidence for supplemental oxygen for symptom management at this threshold of hypoxaemia, whilst others presented anecdotal views that supplemental oxygen was often beneficial for breathlessness in patients with COVID-19 and hypoxaemia.

\section{6) Staff taking care of patients with serious COVID-19 should receive training in optimising clinician-patient communication whilst wearing personal protection equipment \\ Rationale}

Clinician-patient communication is a cornerstone of palliative care. Communication with patients with COVID-19 can be limited by wearing personal protection equipment (PPE). Healthcare professionals' experiences suggest that masks hide facial expressions and muffle voices, restricting the ability to show compassion [25].

\section{Results}

Almost all experts gave a strong $(72.1 \%)$ or conditional $(19.1 \%)$ recommendation for staff taking care of patients with serious COVID-19 receiving training in optimising clinician-patient communication whilst wearing PPE (figure 1f).

Expert experience indicated that PPE was a significant and dehumanising barrier to communication. They felt communication while using PPE was a critically important skill that had to be learned. New ways of showing empathy are needed along with guidance in these skills. One expert described tools developed to improve communication while using PPE, such as flashcards [26].

\section{7) Staff taking care of patients with serious COVID-19 should receive training in online clinician- family communication (while using telephone or video conferencing) \\ Rationale}

Family meetings for patients with COVID-19 are often held online to preserve PPE [27]. Communication and breaking difficult news remotely requires other communication skills in which healthcare professionals usually have no previous training. Healthcare professionals rely on in-person and non-verbal cues to facilitate difficult conversations [28], and may be reluctant to discuss sensitive topics during phone or video consultations [29]. Remote communication is more challenging for people with low literacy or few digital literacy skills, and people with sight or hearing impairment [29]. However, a report from the implementation of palliative care e-family meetings with trained clinicians showed the benefits of these meetings, including satisfied family members [30]. The key elements of remote communication skills have been published [31].

\section{Results}

Most experts provided a strong $(58.8 \%)$ or conditional $(29.4 \%)$ recommendation for staff taking care of patients with serious COVID-19 receiving training in online clinician-family communication (while using telephone or video conferencing) (figure $1 \mathrm{~g}$ ). 
Experts reported the need for a member of the clinical team to communicate remotely with one designated family member or loved one daily. Remote communication skills are seen as essential, but clinicians are often not familiar with remote palliative care conversations.

The lack of evidence base for such training as well as practical concerns were mentioned (table 3).

8) Healthcare professionals trained in providing palliative care should be involved in cases where hospitalised patients with serious COVID-19 have persistent symptoms and concerns despite optimal disease treatment

Rationale

Patients with serious COVID-19 with persistent symptoms and concerns despite optimal disease treatment need optimal symptom management [15]. It has been suggested that symptom burden in dying patients with COVID-19 might be higher than usual in dying patients without COVID-19 [32], resulting in challenges for staff with no or limited experience in palliative care. Therefore, palliative care has an important role for patients with serious COVID-19 and strategies have been implemented to provide in-hospital palliative care $[27,33]$.

\section{Results}

The majority of experts strongly $(63.2 \%)$ or conditionally (23.5\%) recommended that healthcare professionals trained in providing palliative care should be involved in cases where hospitalised patients with serious COVID-19 have persistent symptoms and concerns despite optimal disease treatment (figure $1 \mathrm{~h}$ ).

Experts reported that expertise in palliative care is needed, with involvement of specialist palliative care clinicians varying according to the palliative care skills of the primary clinical team. Specialist palliative care could be invited by the primary clinical team for direct patient/family care, advice to the primary clinical care team, education and development of guidelines. The benefits of involvement of palliative care teams were mentioned, such as: multidisciplinary assessment; the ability to address aspects of care beyond disease treatment, including the management of complex symptoms, support of family and ACP discussions; and support for other healthcare professionals.

9) Healthcare professionals trained in providing palliative care should be involved in cases where patients with serious COVID-19 have persistent symptoms and concerns despite optimal disease treatment treated at home

Rationale

Home palliative care services can provide benefits such as better symptom control [34]. The Association for Geriatric Palliative Medicine (FGPG) recommends the availability of mobile palliative care teams for COVID-19 patients dying at home [35]. Data in COVID-19 are lacking.

\section{Results}

Most experts strongly (51.5\%) or conditionally (33.8\%) recommended the involvement of healthcare professionals trained in palliative care provision in patients treated at home with serious COVID-19 with persistent symptoms and concerns despite optimal disease treatment (figure 1i).

Several experts mentioned that the need for palliative care involvement for patients at home was similar to that described in the hospital setting. Again, the need to involve specialist palliative care depends on the skills of the primary clinical team. The added value of involvement of palliative care professionals to address psychosocial and spiritual needs in the home setting was mentioned.

10) Healthcare professionals providing spiritual care (such as chaplains) should be part of the treatment team of patients with serious COVID-19 with persistent symptoms and concerns despite optimal disease treatment (irrespective of setting, so in the hospital, community or long-term care facilities)

Rationale

Spiritual care is an essential component of palliative care. Spiritual care supports patients and families in facing serious illness and in coping with poor or uncertain prognosis [15]. The COVID-19 crisis has led to fundamental uncertainty in communities, among patients, caregivers and loved ones, and among healthcare professionals. Part of this uncertainty extends beyond healthcare science and into our existential notions of life and death. A rapid review recommended the involvement of spiritual care providers in palliative care for patients with COVID-19 [33]. Attention should also be given to the spiritual care needs of those who are not represented by chaplains or people who are not religious, such as pastoral care workers [15]. 
Results

Most experts strongly (38.2\%) or conditionally (50.0\%) recommended that healthcare professionals providing spiritual care should be part of the treatment team of patients with serious COVID-19 with persistent symptoms and concerns despite optimal disease treatment (figure $1 \mathrm{j}$ ).

Experts mentioned the need for spiritual or existential care beyond that which clinicians can offer, but also suggested that involvement should be dependent on the preferences of the patient and family. Existential care should not be limited to religious care but should include issues in relation to meaning more broadly.

11) Healthcare professionals providing psychosocial care (such as psychologists and social workers) should be part of the treatment team of patients with serious COVID-19 with persistent symptoms and concerns despite optimal disease treatment (irrespective of setting, so in the hospital, community or long-term care facilities)

Rationale

Psychological symptoms such as anxiety and agitation are highly prevalent in dying patients with COVID-19 [6, 19]. Psychosocial interventions in palliative care can relieve emotional and existential distress and improve quality of life [36]. A rapid review recommended that psychosocial care should be provided as part of the palliative care for patients with COVID-19 [33], although data in COVID-19 are not yet available [37].

\section{Results}

Most experts strongly (52.9\%) or conditionally (39.7\%) recommended that healthcare professionals providing psychosocial care should be part of the treatment team of patients with serious COVID-19 with persistent symptoms and concerns despite optimal disease treatment (figure 1k).

Comments provided were that the involvement of psychosocial care should depend on the needs of the patients and families and the existing skills within the primary clinical team.

\section{2) Family members/loved ones should be invited and supported le.g. being provided with PPE if} indicated) to visit the dying patient with COVID-19 in person

Rationale

Because patients with COVID-19 are treated in isolation, family members/loved ones often have no or minimal contact, which may aggravate anxiety and other psychological distress. Moreover, families may be in quarantine or ill themselves [7]. The inability to say goodbye to a family member before death may increase the risk of complicated grief [38]. When family is allowed to visit, PPE might be needed. The value of remote contact between families and dying patients is unknown. Some authors caution against virtual contact between families and dying patients with COVID-19 because it can be too distressing [7]. Other authors do advise remote contact between patients in palliative care and family [39].

\section{Results}

Almost all experts strongly (80.9\%) or conditionally (14.7\%) recommend that family members/loved ones should be invited and supported to visit the patient dying with COVID-19 in person (figure 11).

Most experts acknowledged the importance of a limited number of the closest loved ones being able to visit the dying patient for both patients and families.

\section{3) Family members/loved ones of deceased patients with COVID-19 should be offered} bereavement support by healthcare professionals trained in palliative care or bereavement support

Rationale

Family members/loved ones of deceased patients with COVID-19 might be at increased risk of complicated grief and post-traumatic stress disorder [38, 40]. Several risk factors may be present, such as: a rapid disease trajectory that might have hampered the preparation for death; less social support caused by social isolation; multiple losses due to COVID-19 in one family; feelings of guilt or (self-)blame; and the inability to undertake traditional grieving rituals [38]. Bereavement support is seen as a core component of palliative care. Support to families before and after the death of a patient can positively influence bereavement outcomes [40].

\section{Results}

Most gave a strong (66.2\%) or conditional (23.5\%) recommendation for bereavement support being offered to family members/loved ones of deceased patients with COVID-19 by healthcare professionals trained in palliative care or bereavement support (figure $1 \mathrm{~m}$ ). 
Comments indicated that bereavement risk as well as the need for support will vary and some people will cope through their usual social or community support.

\section{4) Staff caring for patients with serious COVID-19 should be offered psychological support to cope with their experiences \\ Rationale}

During the COVID-19 pandemic, healthcare professionals are experiencing significant distress [41]. Healthcare staff caring for patients with serious COVID-19 may experience secondary traumatic stress; stress caused by observing suffering, and caring for patients dying alone [38, 41]. Challenging ethical decisions such as triaging limited resources may result in moral distress. At the same time, healthcare providers may face personal challenges, such as the decision to isolate themselves from personal support systems out of concern for spreading COVID-19 [38, 41]. During a crisis, attention for self-care may be limited. Adequate supervision and peer support may facilitate self-care, which in turn can overcome accumulated stress and grief in healthcare professionals [38].

\section{Results}

Almost all experts strongly (76.5\%) or conditionally (20.6\%) recommended that staff caring for patients with serious COVID-19 should be offered psychological support to cope with their experiences (figure $1 \mathrm{n}$ ). Comments provided were that healthcare staff should have access to a range of support, depending on their needs. Debriefs within teams were mentioned as a possibility.

\section{Discussion}

This survey provides 14 consensus-based recommendations for palliative care in patients with COVID-19 in the hospital, at home or in other care facilities. Consensus was reached by international experts in different relevant fields, including but not limited to palliative care and respiratory medicine. Given the recent genesis of COVID-19, recommendations are based upon indirect evidence and clinical experience on: ACP; palliative treatment of breathlessness; clinician-patient communication; remote clinician-family communication; palliative care involvement in patients with serious COVID-19; spiritual care; psychosocial care; bereavement care; and support for healthcare professionals. In the absence of evidence-based guidelines, these findings provide consensus guidance for palliative care in COVID-19. The paramount role of palliative care in this pandemic is supported $[3,4]$, but also points to specific challenges and unanswered questions.

ACP was seen as "the first critical step to guide treatment for these seriously ill patients", but the often-rapid disease trajectory, as well as physical absence of family or loved ones, were specific challenges to having these ACP conversations. So, preferably, ACP is initiated much earlier, especially in elderly, chronically ill patients or people with multiple comorbidities, to prepare patients and family for the moment when decisions concerning treatment of COVID-19 should be made. Future data concerning the long-term outcomes of serious COVID-19 are needed to support the process of ACP. Moreover, future studies should explore how to conduct optimal ACP in challenging circumstances like an admission for serious COVID-19.

Palliative treatment of breathlessness seems paramount in COVID-19. Currently, we rely on the commonly known palliative treatment options for breathlessness due to other causes, like opioids, supplemental oxygen in hypoxaemic patients, and benzodiazepines if other treatments fail and where breathlessness is associated with anxiety. Nevertheless, evidence of effectiveness, adverse effects and optimal dosage regimes for opioids and benzodiazepines in COVID-19 are absent. Other physical and psychological symptoms that are also frequently reported by patients with serious COVID-19 $[6,19,20]$ were not included in the present study. Future studies should explore palliative interventions for breathlessness and other symptoms in COVID-19.

To overcome communication barriers in COVID-19, including the need to wear PPE and the physical absence of families and loved ones, healthcare professionals need new skills and experience. Fortunately, training and tools are being rapidly developed to facilitate communication with patients with COVID-19 and their loved ones [26, 30,31,42]. More experience as well as studies exploring the effects of tools and training are needed to optimally support communication skills in palliative care for patients with COVID-19 and their loved ones.

This study supports the value of involvement of healthcare professionals trained in providing palliative care in patients with serious COVID-19 with persistent symptoms and concerns, despite optimal disease treatment in the hospital and at home. The limited availability of palliative care specialists was mentioned as a concern. Nevertheless, not all patients will need to be seen by specialist palliative care clinicians, as 
was also stated by several respondents. Indeed, non-palliative care specialists can be supported to adopt palliative care strategies, through training or consultation for example [42].

\section{Methodological considerations}

These recommendations were developed following the CORE methodology, which has been shown to result in recommendations concordant with those developed using Institute of Medicine-adherent methodology [11]. Nevertheless, several limitations need to be acknowledged. First, although we were able to include experts from 15 countries, we had no respondents from Africa or Asia, but experts from some countries like Denmark, Portugal and The Netherlands were overrepresented. Second, most respondents were physicians; only two nurses and seven allied healthcare professionals participated. Relevant fields of expertise, like family medicine, internal medicine, geriatrics and clinical pharmacology were underrepresented. The wording of questions was unclear to some participants. For example, "healthcare professionals trained in providing palliative care" was interpreted by some respondents as specialist palliative care professionals, and by other respondents as including healthcare professionals with general training in palliative care. Further, defining the population of patients with COVID-19 in need of palliative care was challenging for the author group. We chose the Palliapedia definition of serious disease [12], but other definitions would have been possible. Nevertheless, this definition did not result in comments from participating experts. Finally, we had to limit the survey to 14 possible recommendations. Some aspects concerning palliative care in COVID-19, such as management of agitation, remain unexplored.

\section{Conclusion}

This multi-national task force provides consensus recommendations for the palliative care of patients with COVID-19, concerning: ACP; palliative treatment of breathlessness; clinician-patient communication; remote clinician-family communication; palliative care involvement in patients with serious COVID-19; spiritual care; psychosocial care; bereavement care; and support for healthcare professionals. The fact that 13 out of 14 questions achieved recommendations $>70 \%$ in the first round shows that there is a need to consider palliative care in the treatment of COVID-19 or similar diseases. Future studies are needed to provide empirical evidence for these recommendations.

The goal of consensus guidance is to standardise care, thereby improving outcomes and facilitating research. The suggestions in this document do not constitute official positions of the European Respiratory Society, or the institutions of the task force members. They should not be considered mandates as no suggestion can incorporate all potential clinical circumstances. The suggestions are consensus guidance that should be re-evaluated as evidence accumulates.

Acknowledgements: The authors would like to thank Valerija Arsovski and Alessandra Marguerat from the ERS office in Lausanne (Switzerland) for their logistical support. Moreover, they would like to acknowledge the experts for their time and inputs.

Conflict of interest: M. Ekström has nothing to disclose. D.C. Currow reports that he is an unpaid advisory board member for Helsinn Pharmaceuticals, is a paid consultant and receives payment for intellectual property with Mayne Pharma, and is a consultant with Specialised Therapeutics Australia Pty. Ltd. M.J. Johnson reports institutional payments for consultancy from Mayne Pharma, during the conduct of the study. M. Maddocks has nothing to disclose. A.K. Simonds has nothing to disclose. T. Tonia is an ERS methodologist. K. Marsaa reports personal fees from GlaxoSmithKline, AstraZeneca, Boehringer Ingelheim, Novartis, Roche, Bristol-Myers Squibb, Chiesi Pharma, Kyowa Kirin $\mathrm{AB}$ and Norgine, outside the submitted work. D.J.A. Janssen reports personal fees for lectures from Novartis, Boehringer Ingelheim and AstraZeneca, outside the submitted work.

Support statement: M. Maddocks is funded by a National Institute for Health Research (NIHR) Career Development Fellowship (CDF-2017-10-009) and the NIHR Applied Research Collaboration South London. The views expressed in this publication are those of the author(s) and not necessarily those of the NHS, NIHR or the Department of Health and Social Care.

\section{References}

1 COVID-19 dashboard by the Center for System Science and Engineering (CSSE) at John Hopkins. https:// coronavirus.jhu.edu/map.html Date last accessed: 30 June 2020. Date last updated: 13 July 2020.

2 Flaxman S, Mishra S, Gandy A, et al. Estimating the effects of non-pharmaceutical interventions on COVID-19 in Europe. Nature 2020; 584: 257-261.

3 Radbruch L, Knaul FM, de Lima L, et al. The key role of palliative care in response to the COVID-19 tsunami of suffering. Lancet 2020; 395: 1467-1469.

4 Davies A, Hayes J. Palliative care in the context of a pandemic: similar but different. Clin Med (Lond) 2020; 20: 274-277.

$5 \quad$ Palliative care and the COVID-19 pandemic. Lancet 2020; 395: 1168

6 Lovell N, Maddocks M, Etkind SN, et al. Characteristics, symptom management, and outcomes of 101 patients with COVID-19 referred for hospital palliative care. J Pain Symptom Manage 2020; 60: e77-e81. 
7 Fusi-Schmidhauser T, Preston NJ, Keller N, et al. Conservative management of COVID-19 patients-emergency palliative care in action. J Pain Symptom Manage 2020; 60: e27-e30.

8 National Institute for Health and Care Excellence. COVID-19 rapid guideline: managing symptoms (including at the end of life) in the community. https://www.nice.org.uk/guidance/ng163 Date last updated: 30 April 2020.

9 Association for Palliative Medicine of Great Britain and Ireland. COVID-19 and palliative, end of life and bereavement care in secondary care. Role of the specialty and guidance to aid care. Version 4. https://apmonline. org/wp-content/uploads/2020/04/COVID-19-and-Palliative-End-of-Life-and-Bereavement-Care-20-April-2020-2.pdf

10 Costantini M, Sleeman KE, Peruselli C, et al. Response and role of palliative care during the COVID-19 pandemic: a national telephone survey of hospices in Italy. Palliat Med 2020; 34: 889-895.

11 Wilson KC, Schoenberg NC, Raghu G. Idiopathic pulmonary fibrosis guideline recommendations. Need for adherence to institute of medicine methodology? Ann Am Thorac Soc 2019; 16: 681-686.

12 Pallipedia. Serious illness. https://pallipedia.org/serious-illness/ Date last accessed: 23 May 2020. Date last updated: 7 July 2020.

13 Schoenberg NC, Barker AF, Bernardo J, et al. A comparative analysis of pulmonary and critical care medicine guideline development methodologies. Am J Respir Crit Care Med 2017; 196: 621-627.

14 Rietjens JAC, Sudore RL, Connolly M, et al. Definition and recommendations for advance care planning: an international consensus supported by the European Association for Palliative Care. Lancet Oncol 2017; 18: e543-e551.

15 Bajwah S, Wilcock A, Towers R, et al. Managing the supportive care needs of those affected by COVID-19. Eur Respir J 2020; 55: 2000815.

16 Curtis JR, Kross EK, Stapleton RD. The importance of addressing advance care planning and decisions about do-not-resuscitate orders during novel coronavirus 2019 (COVID-19). JAMA 2020; in press [https://doi.org/10 1001/jama.2020.4894].

17 Janssen DJA, Spruit MA, Schols J, et al. Predicting changes in preferences for life-sustaining treatment among patients with advanced chronic organ failure. Chest 2012; 141: 1251-1259.

18 Janssen DJA, Spruit MA, Schols J, et al. A call for high-quality advance care planning in outpatients with severe COPD or chronic heart failure. Chest 2011; 139: 1081-1088.

19 Sun H, Lee J, Meyer BJ, et al. Characteristics and palliative care needs of COVID-19 patients receiving comfort-directed care. J Am Geriatr Soc 2020; 68: 1162-1164.

20 Keeley P, Buchanan D, Carolan C, et al. Symptom burden and clinical profile of COVID-19 deaths: a rapid systematic review and evidence summary. BMJ Support Palliat Care 2020; in press [https://doi.org/10.1136/ bmjspcare-2020-002368].

21 Ekstrom M, Nilsson F, Abernethy AA, et al. Effects of opioids on breathlessness and exercise capacity in chronic obstructive pulmonary disease. A systematic review. Ann Am Thorac Soc 2015; 12: 1079-1092.

22 Lanken PN, Terry PB, Delisser HM, et al. An official American Thoracic Society clinical policy statement: palliative care for patients with respiratory diseases and critical illnesses. Am J Respir Crit Care Med 2008; 177: 912-927.

23 Simon ST, Higginson IJ, Booth S, et al. Benzodiazepines for the relief of breathlessness in advanced malignant and non-malignant diseases in adults. Cochrane Database Syst Rev 2016; 10: CD007354.

24 Ekstrom M, Ahmadi Z, Bornefalk-Hermansson A, et al. Oxygen for breathlessness in patients with chronic obstructive pulmonary disease who do not qualify for home oxygen therapy. Cochrane Database Syst Rev 2016; 11: CD006429.

25 Earnest M. On becoming a plague doctor. N Engl J Med 2020; in press [https://doi.org/10.1056/NEJMp2011418].

26 Cardmedic. Improving communication in frontline healthcare: communication training tool for staff and students. www.cardmedic.com. Date last accessed: 16 June 2020.

27 Fausto J, Hirano L, Lam D, et al. Creating a palliative care inpatient response plan for COVID-19 - the UW medicine experience. J Pain Symptom Manage 2020; 60: e21-e26.

28 Moore KJ, Sampson EL, Kupeli N, et al. Supporting families in end-of-life care and bereavement in the COVID-19 era. Int Psychogeriatr 2020; in press [https://10.1017/S1041610220000745].

29 Sutherland AE, Stickland J, Wee B. Can video consultations replace face-to-face interviews? Palliative medicine and the Covid-19 pandemic: rapid review. BMJ Support Palliat Care 2020; in press [https://doi.org/10.1136/ bmjspcare-2020-002326].

30 Kuntz JG, Kavalieratos D, Esper GJ, et al. Feasibility and acceptability of inpatient palliative care e-family meetings during COVID-19 pandemic. J Pain Symptom Manage 2020; 60: e28-e32.

31 Chua IS, Jackson V, Kamdar M. Webside manner during the COVID-19 pandemic: maintaining human connection during virtual visits. J Palliat Med 2020; in press [https://doi.org/10.1089/jpm.2020.0298].

32 Turner J, Hodgson LE, Leckie T, et al. A dual-center observational review of hospital-based palliative care in patients dying with COVID-19. J Pain Symptom Manage 2020; 60: e75-e78.

33 Etkind SN, Bone AE, Lovell N, et al. The role and response of palliative care and hospice services in epidemics and pandemics: a rapid review to inform practice during the COVID-19 pandemic. J Pain Symptom Manage 2020; 60: e31-e40.

34 Gomes B, Calanzani N, Curiale V, et al. Effectiveness and cost-effectiveness of home palliative care services for adults with advanced illness and their caregivers. Cochrane Database Syst Rev 2013; 6: CD007760.

35 Kunz R, Minder M. COVID-19 pandemic: palliative care for elderly and frail patients at home and in residential and nursing homes. Swiss Med Wkly 2020; 150: w20235.

36 Warth M, Kessler J, Koehler F, et al. Brief psychosocial interventions improve quality of life of patients receiving palliative care: a systematic review and meta-analysis. Palliat Med 2019; 33: 332-345.

37 Renjun G, Ziyun L, Xiwu Y, et al. Psychological intervention on COVID-19: a protocol for systematic review and meta-analysis. Medicine (Baltimore) 2020; 99: e20335.

38 Wallace CL, Wladkowski SP, Gibson A, et al. Grief during the COVID-19 pandemic: considerations for palliative care providers. J Pain Symptom Manage 2020; 60: e70-e76.

39 Chidiac C, Feuer D, Naismith J, et al. Emergency palliative care planning and support in a COVID-19 pandemic. J Palliat Med 2020; 23: 752-753. 
40 Morris SE, Moment A, Thomas Jd. Caring for bereaved family members during the COVID-19 pandemic: before and after the death of a patient. J Pain Symptom Manage 2020; 60: e70-e74.

41 Brown C, Peck S, Humphreys J, et al. COVID-19 lessons: the alignment of palliative medicine and trauma-informed care. J Pain Symptom Manage 2020; 60: e26-e30.

42 Feder S, Akgun KM, Schulman-Green D. Palliative care strategies offer guidance to clinicians and comfort for COVID-19 patient and families. Heart Lung 2020; 49: 227-228. 